Cuadernos de Lingüística Hispánica $n^{\circ} .23$

ISSN 0121-053X • ISSN en línea 2346-1829

Enero-Junio 2014, pp. 49-65

\title{
Educación bilingüe y pluralidad: reflexiones en torno de la interculturalidad crítica*
}

\author{
DAVID LEONARDO GARCÍA LEÓN** \\ dalgarciale@unal.edu.co \\ JAVIER ENRIQUE GARCÍA LEÓN**** \\ jaegarciale@unal.edu.co
}

Recepción: 2 de junio de 2013

Aprobación: 12 de diciembre de 2013

$\bar{*}^{--}$Artículo de reflexión.

** Magíster en Lingüística de la Universidad Nacional de Colombia., docente de sociolingüística e interpretación discursiva en la Universidad Pedagógica Nacional. Sus principales áreas de estudio son el bilingüismo, la sociolingüística, la criollística y la enseñanza del español como segunda lengua. Sus últimas publicaciones giran en torno de las políticas lingüísticas colombianas.

*** Licenciado en Humanidades: español e inglés y Magíster en Lingüística de la Universidad Nacional de Colombia. Adicionalmente, es miembro del grupo de investigación en Estudios del Discurso de esa misma institución. Docente de producción discursiva y sociolingüística en la Universidad Pedagógica Nacional. Sus áreas de investigación y sus publicaciones giran en torno de las actitudes lingüísticas, la sociolingüística, el análisis crítico del discurso, el bilingüismo y la enseñanza de lenguas. 


\title{
Resumen
}

En este artículo se plantea el problema de la educación bilingüe en la sociedad globalizada. Se describe las características de dicha sociedad y el rol de las lenguas como medio de comunicación en este contexto. Además, se defiende la idea de que la educación bilingüe debe basarse en la perspectiva intercultural crítica, dado que a partir de ella se logra no solo formar en la diversidad y la pluralidad, sino que además permite problematizar situaciones de poder que mantienen relaciones de desigualdad entre los hablantes de diferentes idiomas. Los postulados teóricos que guían estas reflexiones surgen de autores como G rundy (1998), Habermas (1972), Walsh (2010), Freire (1967), García (2009), entre otros. Se espera que este trabajo contribuya a la discusión en torno de la educación bilingüe y las políticas lingüísticas en Colombia.

Palabras clave: educación bilingüe, Interculturalidad crítica, pluralidad, políticas lingüísticas.

\section{Bilingual education and plurality: reflections on critical interculturality}

\begin{abstract}
:
The problem of bilingual education in globalized society is discussed in this article. In this context, the characteristics of society and the role of language as a means of communication are described. Furthermore, this study defends the idea that bilingual education should be based on a critical intercultural perspective. From this idea, it is possible to educate not only in diversity and plurality, but it is also possible to problematize situations of power that maintain relations of inequality among speakers of different languages. The theoretical principles that guide these reflections come from authors such as G rundy (1998), Habermas (1972), Walsh (2010), Freire (1967), G arcía (2009), among others. We hope that this work contributes to the discussion of bilingual education and linguistic policies in Colombia.
\end{abstract}

Key words: Bilingual education, critical interculturality, plurality, linguistic policies. 


\section{Éducation bilingueet pluralité: réflexions autour de l'interculturalité critique}

\section{Résumé}

Dans cet article, on aborde le problème de l'éducation bilingue dans la société globalisée. 0 n décrit les caractéristiques de cette société-ci et le rôle des langues en tant que moyen de communication dans ce contexte. En plus, on défend l'idée qui préconise que l'éducation doit être basée sur la perspective interculturelle critique, dû au fait qu'à partir d'elle on arrive pas seulement à former dans la diversité et la pluralité, mais aussi à problématiser des situations de pouvoir qui permettent de maintenir des rapports d'inégalité entre les usagers des différentes langues. Les postulats techniques qui guident ces réflexions surgissent des auteurs tels que Grundy (1998), Habermas (1972), Walsh (2010), Freire (1967), G arcía (2009), entre autres. On espère que ce travail contribuera à la discussion autour de l'éducation bilingue et les politiques linguistiques en Colombie.

Mots clés: Éducation bilingue, Interculturalité critique, pluralité, politiques linguistiques.

\section{Educação bilinguee pluralidade: reflexoes em torno à interculturalidade crítica}

\section{Resumo:}

O artigo apresenta o problema da educação bilíngue na sociedade globalizada. D escreve as caracteństicas de dita sociedade e o papel da língua como meio de comunicação neste contexto. Alem disso, se defende a ideia que a educação bilíngue deve fundamentarse na perspectiva intercultural crítica, porque a partir dela se logra não só formar na diversidade e na pluralidade, mas também permite problematizar situações de poder que mantém relações de desigualdade entre os falantes de diferentes línguas. Os postulados teóricos que guiam ditas reflexões surgem de autores como G rundy (1998), Habermas (1972), Walsh (2010), Freire (1967), G arcía (2009), entre outros. Espera-se que o trabalho contribua na discussão da educação bilíngue e as políticas linguísticas na Colômbia.

Palavras Chave: Educação bilíngue, interculturalidade crítica, pluralidade, políticas inguísticas. 


\section{Introducción}

En los últimos años, ha existido una intensa reflexión sobre la necesidad de aprender lenguas extranjeras y sobre la reivindicación de losidiomas de grupos minoritarios. Esto se ve reflejado, por ejemplo, en la creación e implementación de políticas lingüísticas en Colombia como el Plan Nacional de Bilingüismo (PNB) y la Ley de lenguas. Estos dos documentos, aunque con objetivos distintos, se enmarcan en los discursos que, por un lado, propagan la necesidad de manejar diferentes idiomas en un mundo globalizado y, por el otro, sostienen que es importante respetar la diversidad lingüística. Este fenómeno ha conducido a la creación e implementación de diferentes programas de educación bilingüe dependiendo de las comunidades a las que se dirigen. En este ensayo, entonces, se intentará establecer cuál es el sentido de dicha educación en la actualidad, argumentando que la educación bilingüe debería ser esencialmente crítica y pluralista, es decir, esta debe servir a la justica social.

Para alcanzar el objetivo planteado, el texto se organiza de la siguiente manera. En primer lugar, se explica por qué es importante que la educación bilingüe se centre en el entendimiento de la pluralidad en el momento histórico actual; segundo, se discute la educación bilingüe a la luz de los intereses humanos propuestos por Habermas (1972). En el tercer apartado, se sostendrá que la educación bilingüe, además de intercultural, debe ser crítica. En este último apartado se muestra la necesidad de dicha perspectiva a través del estudio que autores como García y García (2012), Usma (2009) y G uerrero (2010) realizan de algunas políticas lingüísticas colombianas y de los aportes de Ferrão (2010) en cuanto a la relación educación intercultural y democracia.

\section{Educación bilingüey globalización}

Entender por qué la educación bilingüe debe ser una educación centrada en la pluralidad requiere entender cómo los procesos históricos en que vivimos han modificado las prácticas de uso del lenguaje. D e acuerdo con G arcía (2009), 
en el siglo XXI se ha presentado un crecimiento económico y un nuevo orden socio-político que ha afectado la forma en que se usa el lenguaje. Los aspectos económicos han llevado a que se den cambios a nivel de las poblaciones y al surgimiento de la comunicación transnacional. En relación con el primer elemento, es importante mencionar que los fenómenos de migración han generado diversos tipos de contacto cultural y lingüístico, amplificando la presencia del bilingüismo en la medida en que los inmigrantes, refugiados, comerciantes, entre otros, necesitan comunicarse y entrar en la esfera de la información (pp. 26- 27). Por su parte, el surgimiento de la comunicación transnacional se debe al desarrollo de nuevas tecnologías como la internet. La red ha permitido el aumento del contacto lingüístico independientemente del lugar geográfico en que se encuentran los usuarios de las lenguas. La diferencia entre las prácticas de uso del lenguaje en los siglos anteriores y la actualidad radica en que los ámbitos donde utilizamos la habilidad de comunicar se han ampliado, por ello el bilingüismo es un elemento central en el proceso de comunicación (p. 29). Ahora, más que nunca, estamos en constante contacto con lenguas y culturas; sin embargo, para poder moverse en estos nuevos escenarios de comunicación no solo se requiere de un saber lingüístico, sino también de un saber cultural.

Los cambios culturales anteriormente mencionados no se limitan a la esfera económica. En el nivel social, el constante contacto cultural, producto de la globalización, también ha influido en aspectos como la identidad. D e acuerdo con García (2009), las identidades nacionales juegan un rol importante en este mundo sin límites económicos. La eliminación de los límites geográficos, por medio del libre comercio y las nuevas tecnologías de la información, es lo que origina la denominada "glocalización", es decir, la posibilidad de que la identidad nacional esté inmersa en lo global y viceversa (p. 30). D e este modo, las lenguas son factores esenciales en los procesos de entendimiento cultural, pues a través de ellas llevamos nuestro mundo individual al global y nos apropiamos de lo global como individual. La identidad deja de ser un elemento fijo, pasa a hacer un elemento híbrido mediado por la(s) lengua(s).

Sumado a lo anterior, los individuos local-globales deben saber cómo adaptarse y actuar en un determinado ambiente cultural. Siguiendo a Truscott de Mejia (2012), aquellas personas que entran en contacto con diferentes culturas deberían valorar la tensión que dicho contacto genera, entendiéndolo como un mecanismo que les permite hacer parte de lo universal. Es en este aspecto donde la educación bilingüe tiene un rol fundamental, pues es a partir de ella que se 
puede entender la tensión que generan los encuentros culturales. Apropiarse de un segundo o tercer código lingüístico permite, entonces, apreciar dichas tensiones de primera mano. Sin embargo, no debe limitarse a la simple apreciación, pues la comprensión cultural debería derivar en el logro de un grado más alto de comprensión que trascienda no solo nuestra propia particularidad sino, también, la del otro (p.16). La educación bilingüe debe, desde este enfoque, preparar para la diversidad, para los diferentes aspectos sociales, políticos y económicos en los que se ven inmersos los individuos en un mundo globalizado, por ello, la educación bilingüe tiene sentido si es una educación multicultural, es decir, una educación en los siguientes términos:

La preparación para las realidades sociales, políticas y económicas que los individuos experimentan en los encuentros humanos, y que son culturalmente diversos y complejos. Esta preparación proporciona los procesos por medio de los cuales un individuo desarrolla la competencia para percibir, crecer y comportarse en situaciones culturales diferentes (Banks, 1981, citado por Muñoz, 1995, p. 229).

Sin embargo, cabe aclarar que la educación bilingüe desde un enfoque multicultural en pro de la diversidad, no debe limitarse a la simple percepción y aceptación del otro. Siguiendo a Muñoz (1995, p. 230), la educación más que multicultural debe ser intercultural, esto es, se debe educar a los seres humanos en el conocimiento, comprensión y respeto por las diferencias culturales en que ellos viven. Dicha educación, debería, desde esta perspectiva, considerar los códigos lingüísticos como el medio para transformar las prácticas sociales. Las lenguas, en la educación bilingüe, no serían simples sistemas para comprender al otro, pues tienen un potencial superior: permitir el diálogo para la resolución de conflictos y creación de consensos. Una educación bilingüe intercultural tiene como fin formar sujetos capaces de convivir en la pluralidad (Brayam, 1997, p. 32). En resumen, la educación bilingüe tiene sentido si no se limita al aprendizaje de elementos lingüísticos y discursivos, pues en un momento histórico marcado por la diversidad, la competencia intercultural es un elemento fundamental en la educación:

D esde este punto de vista, hoy la interculturalidad también supone apertura frente a las diferencias étnicas, culturales y lingüísticas; a la aceptación positiva de la diversidad; al respeto mutuo; a la búsqueda de consenso y, paralelamente, al reconocimiento y aceptación del disenso y, actualmente, a la construcción de nuevos modos de relación social y de más democracia (Ferrão Candau, 2010, p. 337). 


\section{La educación bilingüey los intereses humanos}

D esde la perspectiva intercultural, enmarcada en un contexto global, vale la pena analizar el papel de las lenguas en la educación bilingüe. Si dicho tipo de educación tiene como objetivo formar en la aceptación del otro y en la construcción de consensos en las relaciones humanas ¿cuál es el papel de las lenguas y cómo deben ser tratadas en este tipo de educación? Para responder estos interrogantes es necesario partir de la teoría de los intereses humanos propuesta por Habermas (1972) y retomada por G rundy (1998). En primer lugar, la acción de hablar es un elemento determinante en los intereses humanos, pues es a partir de ella que el sujeto se hace miembro de una sociedad y entiende las orientaciones fundamentales de la especie humana (Grundy, 1998, p. 25). En este sentido, las acciones que realizan los seres humanos, discursivas o no, están determinadas por una serie de intereses fundamentales que, para Habermas (1972), pueden clasificarse en tres grupos: técnico, práctico y emancipatorio.

El interés técnico se basa en controlar y gestionar el medio ambiente (Grundy, 1998, p. 27). D esde esta perspectiva, por ejemplo, la ciencia tiene como objetivo controlar la naturaleza por medio de la investigación experimental y generar reglas a partir de las cuales los seres humanos puedan actuar. Las acciones bajo este tipo de interés son acciones instrumentales, es decir, están orientadas a utilizar los recursos como herramientas en pro de la consecución de un fin preestablecido. Bajo este interés, el aprendizaje de una lengua se limitaría a la adquisición de una herramienta que nos permita conseguir otros objetivos; la lengua es, entonces, un mero instrumento. Este sería el caso, por ejemplo, de los programas bilingües de transición, en donde la lengua materna es solo un instrumento para adquirir algunos saberes, entre estos la lengua mayoritaria. La diversidad lingǘstica, desde esta perspectiva, no es tenida en cuenta, pues no importa que las lenguas sean un eje transmisor de culturas, lo que interesa es su utilización para lograr fines específicos como aprender a leer y escribir y desarrollar habilidades matemáticas. Una educación bilingüe en pro de la pluralidad no puede plantearse desde este enfoque, en tanto que las lenguas no son simples herramientas para lograr fines establecidos, son, por el contrario, parte fundamental de los procesos culturales en que se utilizan.

Por su parte, el interés práctico se basa en comprender el medio ambiente para interactuar con él (G rundy, 1998). Dicha interacción se fundamenta en la comprensión de la realidad, es decir, es necesario conocer el marco socio-histórico con el fin de comprender cuál es la mejor forma de interactuar en dicho medio. 
De este modo, luego de que se comprende el contexto en que se encuentran los individuos se llega a acuerdos sobre cómo actuar en ese momento histórico. Sin embargo, la comprensión del medio, no necesariamente significa su problematización. Se comprende la realidad para tomar decisiones pero no para cambiarla. D esde este enfoque, la educación bilingüe, se limitaría a entender la existencia de la diversidad lingǘstica, trataría de comprenderla y a partir de dicha interpretación se formarían a los sujetos para vivir en las sociedades multiculturales. Aquí, el aprendizaje de un segundo o tercer código lingüístico serviría para comprender que existen otras culturas y poder relacionarse con ellas.

No obstante, este enfoque, al responder simplemente a la situación histórica, no permite la construcción de nuevos modos de relación social. Al no problematizar la realidad, se limita a su entendimiento y aceptación, perpetuando injusticias sociales y formas dominantes de entender la realidad. De acuerdo con Freire (1967, p. 37), no problematizar la realidad es aceptar la dominación, es convertirse en dominador. Así, una educación bilingüe que no cuestione la realidad lingüística y las relaciones de poder que se dan entre las lenguas sería una falsa educación hacia la pluralidad pues se limitaría a la aceptación del otro, perpetuando diferencias de poder (Bourdieu, 1991, p. 20). La verdadera educación bilingüe, como se mencionó, debe propiciar la construcción de consensos entre culturas que permitan vivir en equidad en el mundo globalizado; no se puede olvidar que la pluralidad es un asunto de grupos sociales que entran en relación por medio de las lenguas.

En tercer interés, el emancipador, se basa en acciones autónomas y responsables que parten de decisiones informadas por cierto tipo de saber (Grundy, 1998). Para ello, es necesario generar teorías críticas que expliquen cómo funciona la realidad. Dichas teorías no solo deben entender el contexto sociohistórico dónde se encuentra el individuo, sino informar los juicios para denunciar y cuestionar dicho contexto. De este modo, el interés emancipador tiene como fin último el cambio de la realidad por medio de su problematización:

Mientras los otros dos intereses se ocupan del control y de la comprensión, respectivamente, el emancipador se ocupa de la potenciación, o sea, de la capacitación de individuos y grupos para tomar las riendas de sus propias vidas de manera autónoma y responsable. El interés cognitivo emancipador puede definirse de este modo: un interés fundamental por la emancipación y la potenciación para comprometerse en una acción autónoma que surge de 
intuiciones auténticas, criticas, de la construcción social de la sociedad humana (Grundy, 1998, p. 38).

La educación bilingüe vista desde el interés emancipatorio es una educación intercultural que no solo busca el reconocimiento del otro, sino liberarse con él. Esto es, la educación serviría para modificar situaciones de opresión y dominación, pues a partir de las teorías criticas se problematiza el mundo, con el objetivo de cambiarlo. Las lenguas serían fundamentales en dicho proceso en tanto que permiten llegar a consensos sobre los problemas sociales existentes y ayudan a la creación de métodos para solucionarlos. Así, el aprendizaje de un código lingǘstico debería llevar consigo el entendimiento critico de la realidad, esto significa, por ejemplo, entender el mercado lingǘstico que se ha venido desarrollando entre lenguas mayoritarias y minoritarias, entre lenguas de poder y lenguas subordinadas, entre lenguas de "prestigio" y "dialectos", entre otros elementos. Solo a través de la lectura crítica de dicha realidad es que verdaderamente se puede hacer una educación en pro de la pluralidad, pues entender al otro no solo es reconocerlo, es ser capaz de liberarse con él a través del cambio cultural. La educación bilingüe emancipadora entonces "pretende ser una propuesta ética y política con el objetivo de construir sociedades democráticas que articulen la igualdad y el reconocimiento de las diferentes culturas" (Ferrão Candau, 2010, p. 338).

La educación bilingüe desde la perspectiva emancipadora debe ceñirse, entonces, a dos principios: la justicia social y la práctica social (G arcía, 2009, p. 318). El primero se refiere a la aceptación de los diversos grupos lingüísticos y sociales que interactúan, es decir, dado que en la educación bilingüe entran en juego dos o más lenguas y culturas, es necesario que estas sean tratadas con el mismo respeto e igualdad. Tanto los hablantes de lenguas minoritarias como los de lenguas mayoritarias tienen que ser considerados como individuos capaces de reflexionar sobre sus prácticas sociales (G arcía, 2009, p. 319). La educación bilingüe bajo este principio buscará que los miembros de su comunidad sean tolerantes frente a las lenguas y culturas con que trabajan.

De este modo, el eje del principio de justicia social es la equidad. Equidad en el tratamiento de los estudiantes, de sus lenguas y de sus culturas. D ada la diversidad, la equidad se debe brindar, principalmente, al nivel de la participación. Los miembros deben ser libres para dialogar y problematizar su realidad. Así, no existirán lenguas de "prestigio" ni diglosia educativa permitiendo que sus usuarios utilicen las lenguas libremente y en pro del consenso social. Sumado a lo anterior, 
la educación bilingüe en pro de la pluralidad debe fomentar el desarrollo de la conciencia multilingüística: "bilingual pedagogy must not only focus on how language works and how students use and learn languages, but also on the understanding on the social, political and economic struggles surrounding the use of two languages-what has become known as critical language awareness" (García, 2009, p. 320).

En cuanto a la práctica social, esta se basa en posicionar el aprendizaje como el resultado de acciones culturales colaborativas (G arcía, 2009, p. 323). A prender una lengua es hacer parte de una comunidad con prácticas lingǘsticas definidas, por ello, su aprendizaje debe estar basado en interacciones comunicativas significativas que se realizan a través del diálogo. Cabe mencionar que dichas interacciones no se limitan al aprendizaje de un código lingüístico, pues además tienen como propósito aprender sobre la forma en que el otro entiende el mundo, su cultura y sus condiciones sociales. A través del diálogo se llega a consensos sociales y a comprender las formas de la realidad que se quieren cambiar. Es en el diálogo donde las lenguas tienen un papel fundamental, pues median entre los saberes de cada individuo y la construcción de nuevas realidades.

\section{H acia una educación intercultural bilingüe crítica}

Como se evidenció en el apartado anterior, la educación bilingüe debe asumir una perspectiva intercultural emancipatoria o crítica. En esta sección se ahondará en las características de este tipo de educación. Para ello, se usan los postulados de Tubino (2005) y Walsh (2010) sobre interculturalidad y se presentan las criticas que algunos investigadores colombianos han hecho a las políticas lingüísticas del país.

En primer lugar, para Walsh (2010), el concepto de interculturalidad no se escapa, como ningún otro concepto, a cargas sociopolíticas. Así, entender las marcas ideológicas que tiene este concepto permite plantear perspectivas más críticas y reflexionar sobre el uso que hacemos del mismo. De esta manera, la interculturalidad se ha concebido desde tres perspectivas: relacional, funcional y crítica (p. 76). La primera se fundamenta en el hecho de que, en regiones como Latinoamérica, la relación entre comunidades con culturas diferentes siempre ha existido. Esto significa que la interculturalidad no es entendida como una situación de conflicto, sino natural al encuentro de comunidades:

Se asume que la interculturalidad es algo que siempre ha existido en América

Latina porque siempre ha existido el contacto y la relación entre los pueblos 
indígenas y afrodescendientes, por ejemplo, y la sociedad blanco-mestiza criolla, evidencia de lo cual se puede observar en el mismo mestizaje, los sincretismos y las transculturaciones que forman parte central de la historia y "naturaleza" latinoamericana-caribeña (p. 77).

Como se muestra, la interculturalidad se entiende como un proceso natural desligado de las relaciones de poder que se generan con el encuentro entre comunidades. Esta perspectiva cae en el mantenimiento de relaciones de dominación al entender como natural los contactos culturales. D e esta manera, para Walsh (2010) : "el problema con esta perspectiva es que, típicamente, oculta o minimiza la conflictividad y los contextos de poder, dominación y colonialidad continua en que se lleva a cabo la relación" (p. 77). Por lo tanto, asumir la educación bilingüe intercultural desde esta perspectiva mantendría las relaciones de dominación que se establecen entre las comunidades lingǘsticas minoritarias y aquellas que ostentan el poder. Esta perspectiva también mantiene problemas estructurales como la pobreza y el racismo al dejar de lado los aspectos políticos, económicos y epistemológicos que direccionan los encuentros culturales.

Para entender las dos perspectivas restantes, funcional y crítica, es necesario retomar los postulados de Tubino (2005). Para este autor, diferentes programas de educación bilingüe en América Latina asumen la interculturalidad desde una visión que es compatible con las reglas de juego del capitalismo tardío (p. 2), esto significa que no se cuestiona el contexto sociocultural en el que las políticas educativas toman lugar. A esta forma de entender la interculturalidad se le denomina perspectiva funcional, pues como su nombre lo indica, entraa funcionar en la estructura política, económica y social establecida. Esta perspectiva acepta la diferencia, busca promover el diálogo intercultural y alcanzar la convivencia tolerante, pero cuidándose de no cuestionar la lógica neoliberal:

En este sentido, el reconocimiento y el respeto a la diversidad cultural se convierten en una nueva estrategia de dominación, que apunta no a la creación de sociedades más equitativas e igualitarias, sino al control del conflicto étnico y la conservación de la estabilidad social con el fin de impulsar los imperativos económicos del modelo (neoliberalizado) de acumulación capitalista, ahora "incluyendo" a los grupos históricamente excluidos en su interior (Walsh, 2010, p. 78).

De esta manera, tenemos dos momentos importantes de la educación bilingüe. Primero, uno en donde no había respeto por la cultura y lengua del otro y en donde los programas educativos eran aculturizadores y sustractivos. 
En ese momento, la diferencia cultural y lingüística era vista como problema y no era reconocida (García, 2009, p. 14). El segundo momento, se caracteriza por el respeto por dichas diferencias, pero con el fin de que estas no se tornen conflictivas con el modelo social y económico dominante. Este tipo de educación bilingüe es intercultural funcional y se encuentra en boga en la actualidad, especialmente, pero no solo en los programas educativos para comunidades minoritarias. Ejemplo de esto son las reformas constitucionales en la década de los noventa según Walsh (2010); pues allí se reconocen a los estados como multilingües y pluriculturales con el fin de apaciguar las luchas sociales que se venían dando, pero sin una modificación sustancial de la situación económica y social en que vivían las comunidades minoritarias.

El caso colombiano también es característico de esta lógica funcional en cuanto a la educación intercultural bilingüe. En la actualidad, los programas de educación bilingüe en este país se pueden clasificar, siguiendo a $\mathrm{G}$ arcía y $\mathrm{G}$ arcía (2012, p. 14) y a Mejía (2011, p. 59), en dos grandes grupos: la educación bilingüe de élite y la etnoeducación. Esta última es implementada en las comunidades indígenas y busca, por un lado, dar reconocimiento a sus lenguas y culturas y, por el otro, permitirles el acceso a la sociedad mayoritaria. Este tipo de educación, sin embargo, no ha conducido a una mejora en las condiciones económicas y sociales de los indígenas, en la medida en que se inscribe en la lógica funcional intercultural. Siguiendo a Freire (1967, p. 195), la educación intercultural funcional no es una educación problematizadora que ponga en tela de juicio la cultura en que se enmarcan las prácticas sociales. Este tipo de educación continúa naturalizando un fenómeno que es puramente cultural, la desigualdad social.

Esta perspectiva funcionalista puede también explicarse desde los intereses técnico, práctico y emancipatorio anteriormente explicados. El interés práctico, mitatis mitandis correspondería a la lógica funcional de la educación bilingüe aquí descrita, pues este se define como "un interés fundamental por comprender el ambiente mediante la interacción, basado en una interpretación consensuada del significado" (Grundy, 1998, p. 32). De esta manera, los sujetos buscan comprender el contexto en el que se encuentran mediante la interacción con el otro sin buscar un cambio real de las situaciones en las que se encuentran. Un ejemplo característico de esto es la implementación de políticas lingüísticas en torno de la enseñanza de lenguas extranjeras en Colombia. Para autores como Guerrero (2010), políticas como el Plan nacional de bilingüismo (PNB) se fundamentan en el hecho de que en un mundo globalizado se vive 
interculturalmente; sin embargo, no se cuestionan ni se tienen en cuenta las condiciones sociales y económicas en las que estas políticas se implementan. Para ella, en Colombia:

The discourse of equality has influenced language policy decisions under the premise that speaking English gives equal opportunities particularly in the economic field, but this ideology blurs the recognition of the inequality to the access of ways to learn English. There is a big difference between attending public under-funded and overcrowded schools and attending elite private schools (Guerrero, 2010, p. 301).

D e esta manera, políticas como el PNB usan conceptos como la equidad para ocultar los conflictos que viven los sujetos. De nuevo, partimos de una perspectiva "incluyente" que no cuestiona de fondo las realidades materiales e inmateriales en las que se encuentran los individuos. A esta crítica al PNB, se adhiere Usma (2009, p. 134) cuando sostiene que dicha política produce de fondo una estratificación lingüística. Por lo tanto, aunque el PNB se manifiesta como una política que permitirá a los ciudadanos del común interactuar con otras culturas a través del inglés, de fondo genera una clasificación de las lenguas basándose en su utilidad en el mercado lingüístico, pues se considera que solo el inglés es lengua de progreso y cultura, las lenguas minoritarias son devaluadas y caracterizadas como inapropiadas para acceder al mundo globalizado. Así, este tipo de políticas terminan manteniendo las relaciones de dominación y desigualdad aunque sostengan que su perspectiva es intercultural.

Como se ha venido mostrando, las perspectivas relacional y funcional en la educación intercultural bilingüe no tocan de fondo los problemas estructurales en los que se enmarcan los ciudadanos, sino que por el contrario, mantienen, muchas veces, las relaciones de dominación en la que estos se encuentran. Por esto, es necesario que el sentido de la educación bilingüe sea crítico y mejore las condiciones sociales de las personas. Esto significa que, siguiendo a G arcía (2009, p. 12), la educación bilingüe no debe limitarse a la convivencia de dos lenguas en la escuela, sino que debe ampliar su perspectiva y buscar la justicia social. Por lo tanto, este tipo de educación debe ser intercultural crítica atendiendo a la propuesta de Walsh (2009), quien considera que lo más importante de la interculturalidad crítica:

Es su entendimiento, construcción y posicionamiento como proyecto político, social, ético y epistémico -de saberes y conocimientos-, que afirma la necesidad de cambiar no solo las relaciones, sino también las estructuras, condiciones y 
dispositivos de poder que mantienen la desigualdad, inferiorización, racialización y discriminación ( p. 79).

La perspectiva que se defiende aquí es emancipadora atendiendo a la propuesta de Grundy (1998, p. 39). Para esta autora, como se mencionó, el interés que debe guiar un currículo escolar debe ser emancipador; esto significa que cualquier ejercicio educativo debe hacer conscientes a los sujetos de las relaciones deformadas de la realidad en la que se encuentran, permitiéndoles crear un mundo más justo y equitativo en donde prime el consenso y se abandonen las relaciones desiguales. Esto no es posible en otros tipos de educación en la medida en que no se problematiza el mundo y se asume la realidad como inmodificable. En la perspectiva crítica, la educación bilingüe asume que las condiciones de las lenguas, por ejemplo, tienen su origen en tensiones ideológicas; es decir, el hecho de que existan, por ejemplo, lenguas con mayor prestigio que otras parte de las fuerzas de poder de los ciudadanos que las hablan y no es un estado natural. Además, entiende que las lógicas dominantes crean conceptos que parecen neutros y que deben ser cuestionados como es el caso de la interculturalidad funcional o práctica.

Es importante ahondar en las características de este tipo de educación bilingüe intercultural. En primer lugar, la educación intercultural crítica parte siempre de un diálogo contextualizado. Esto quiere decir que cualquier ejercicio educativo que se enmarque en esta perspectiva parte de las experiencias de sus participantes y no puede ser el simple traslado de perspectivas ajenas a situaciones concretas. Además, la interculturalidad debe ser entendida, siguiendo a Ferrão (2010), como un fenómeno de todos y no desde la inclusión del otro considerado como diferente, pues de lo contrario se caería en la lógica funcional donde se reconoce al otro, pero no se modifica el accionar de los sujetos que ostentan el poder:

La educación intercultural se dirigía exclusivamente a los grupos subalternizados, generalmente étnico-raciales y sobre todo a los indígenas y, menos frecuentemente, a los afroamericanos. Son estos grupos los "otros", los "diferentes" y los que, desde la óptica de la interculturalidad funcional, deben ser integrados a la sociedad nacional. Sin embargo, [... ] se ha venido afianzando la postura de que la interculturalidad debe ser trabajada por todos los agentes sociales, si realmente queremos que se vuelva una característica de la sociedad como un todo, en su proceso de construcción democrática (Ferrão Candau, 2010, p. 8). 
Otra característica que debe resaltarse de este tipo de educación es que tiende a desarrollar una conciencia crítica y no se limita a una educación comunicativa (Roux, 2012, p. 60). Esto es importante en la medida en que la comunicación se encuentra inmersa en un conjunto de ideas que deforman la realidad y que en términos de G rundy (1998, p. 155) deben ser cuestionados a través de teoremas críticos. Así, la educación bilingüe crítica combina reflexión y práctica, pues busca develar los discursos hegemónicos que presentan la realidad como inmodificable y a la vez trabaja el cambio de las desigualdades. Se podría afirmar que la educación intercultural bilingüe crítica es, entonces, una reflexión en torno de las prácticas lingüísticas y sociales de las comunidades y lenguas que entran en contacto con el fin de establecer relaciones marcadas por la justicia social y el respeto por el otro.

\section{Conclusiones}

Como se observó, la educación bilingüe en un mundo globalizado adquiere sentido si su objetivo es formar en la pluralidad y el consenso social. Para ello, dicho tipo de educación no puede verse como la suma de dos o más códigos lingüísticos. La educación bilingüe debe plantearse una meta más amplia: "to educate generally, meaningfully, and for tolerance and apreciation of diversity" (García, 2009, p. 6). Para ello, no se puede entender la educación bilingüe a partir de un interés técnico ni práctico. Este tipo de educación debe ser emancipador, debe formar a los sujetos para que decidan frente a sus vidas a partir del dialogo intercultural. El sentido de la educación bilingüe está en entender al otro para llegar a consensos basados en la justicia y la práctica social. Adicionalmente, este tipo de educación debe cuestionar las lógicas que mantienen desigualdades sociales y la estratificación de culturas y lenguas. Dado que la interculturalidad como concepto puede ser útil a discursos hegemónicos, es necesario plantear una educación bilingüe intercultural crítica que tenga como ideal la desarticulación de las relaciones de poder e injusticia en las que se enmarcan las lenguas y las culturas. 


\section{Referencias bibliográficas}

Bourdieu, P. (1991). Languageand symbolic poner. Cambridge: Polity.

Brayam, M. (1997). Teading and assesing interaitural communicative comptence Clevendon: Multilingual matters.

Ferrão Candau, V. M. (2010). Educación intercultural en América Latina: distintas concepciones y tensiones actuales. En: revista Estudios Peedagógicos N․ 36 (2), p.p. 333-342.

Freire, P. (1967). Peelaggóa dd qpimida Bogotá: América Latina.

Garcia, J., \& G arcia, D. (2012). LinguisticPdiyin Cdonkia: TeadingEngishas theDaminant Languagevs Teahing of San AndresianEngish Crele Paper presented at the 19th Biennial Conference of the Society for Caribbean Linguistics (SCL). The College of the Bahamas. Nassau, Bahamas.

García, O. (2009). Bilingual Education in the21st Century: A gdbal perspetive Singapore: Wiley-Blackwell.

Grundy, S. (1998). Producto o praxis dd amiaulum España: Ediciones Morata, S.L.

Guerrero, C. (2010). The Portrayal of EFL Teach ers in Official Discourse: The Perpetuation of Disdain. En: revista PROFILE, No. 12(2), p.p. 33-49.

Habermas, J. (1972). Knowłeegeand human inteets London: Heinemann.

Mejia, A.-M. d., \& Montes, M. E. (2011). El bilingüismo y el multilingüismo en Colombia: Consideraciones acerca de su valor y promulgación. In E. Yoko \& J. Santana (Eds.), Bilinguismos suljeivação eidanificaçães nas palas línguas matemas e estrangiras (pp. 55-81). Brasil: Pontes Editores.

Muñoz, A. (1995). La educación inercultural hoy. En: revista Didádtica No.7, p.p. 217-249. 
Roux, R. (2012). Enseñanza de lenguas extranjeras y formación ciudadana: desarrollando competencias para la globalización cultural. En: revista Rexista Intemacional Magisteio, No. 58, p.p. 58-64.

Truscott de Mejia, A.-M. (2012). Bilingualism, opening to the world and appreciating pluralism and difference. En: revista Reista Intemaaional Magisteio No. 58, p.p. 14-23.

Tubino, F. (2005). Lainteculturalidadoćticacomppryetoéticopdítica Paper presented at the Encuentro continental de educadores agustinos., Lima. Recuperado de http:/ / oala.villanova.edu/ congresos/ educación/ limaponen-02.html

Usma, J. (2009). Globalization and Language and Education Reform in Colombia: A Critical O utlook. En: revista Íkala, reistadelengrajeyaittura, No. 14(22), p.p.19-42.

Walsh, C. (2010). Interculturalidad crítica y educación intercultural. In J. Viaña, L. Tapia \& C. Walsh (Eds.), Construyendo Interculturalidad Crítica (pp. 7596). Bolivia: Instituto Internacional de Integración del Convenio Andrés Bello. 